\title{
Cholesterol Synthesis Is Associated with Hepatic Lipid Content and Dependent on Fructose/Glucose Intake in Healthy Humans
}

\author{
Guenther Silbernagel, ${ }^{1}$ Dieter Lütjohann, ${ }^{2}$ Juergen Machann, ${ }^{3}$ \\ Sabrina Meichsner, ${ }^{2}$ Konstantinos Kantartzis, ${ }^{1}$ Fritz Schick, ${ }^{3}$ \\ Hans-Ulrich Häring, ${ }^{1}$ Norbert Stefan, ${ }^{1}$ and Andreas Fritsche ${ }^{1}$ \\ ${ }^{1}$ Division of Endocrinology, Department of Internal Medicine, Diabetology, Nephrology, Vascular Disease, and Clinical Chemistry, \\ Eberhard-Karls-University Tübingen, Otfried-Müller-Straße 10, 72076 Tübingen, Germany \\ ${ }^{2}$ Institute of Clinical Chemistry and Clinical Pharmacology, University Clinic Bonn, Sigmund-Freud-Straße 25, 53127 Bonn, Germany \\ ${ }^{3}$ Section on Experimental Radiology, Department of Diagnostic Radiology, Eberhard-Karls-University Tübingen, \\ Hoppe-Seyler-Straße 3, 72076 Tübingen, Germany
}

Correspondence should be addressed to Andreas Fritsche, andreas.fritsche@med.uni-tuebingen.de

Received 28 July 2011; Accepted 8 September 2011

Academic Editor: Faidon Magkos

Copyright (๑) 2012 Guenther Silbernagel et al. This is an open access article distributed under the Creative Commons Attribution License, which permits unrestricted use, distribution, and reproduction in any medium, provided the original work is properly cited.

\begin{abstract}
Visceral obesity and fatty liver have been related to high synthesis and low absorption of cholesterol. This study aimed to investigate the associations of cholesterol metabolism with liver and visceral fat content in healthy humans. Another objective was to explore the effects of very-high-fructose and very-high-glucose diets on cholesterol homeostasis. We report on a cohort of 20 people (12 males, 8 females; age $30.5 \pm 2.0$ years; body mass index $25.9 \pm 0.5 \mathrm{~kg} / \mathrm{m}^{2}$ ) who completed a four-week dietary intervention study. Between the baseline and the followup examination the study participants in addition to a balanced weight-maintaining diet received $150 \mathrm{~g}$ of either fructose or glucose per day. Visceral and liver fat were measured with magnetic resonance (MR) imaging and ${ }^{1} \mathrm{H}-\mathrm{MR}$ spectroscopy, respectively. Cholesterol absorption and synthesis were estimated from the serum noncholesterol sterol concentrations. Performing cross-sectional analyses the lanosterol and desmosterol to cholesterol ratios were positively correlated with visceral and liver fat content (all $P<.03$ ). The lathosterol to cholesterol ratio decreased in response to high-fructose diet $(P=.006)$ but not in response to high-glucose diet. To conclude, visceral and liver fat content are associated with cholesterol synthesis in healthy humans. Furthermore, cholesterol synthesis appears to be dependent on fructose/glucose intake.
\end{abstract}

\section{Introduction}

Serum cholesterol is either derived from intestinal absorption or from endogenous synthesis [1]. The individual balance of cholesterol absorption and synthesis is highly heritable [2]. The ATP-binding cassette transporters G5 and G8 (ABCG5/8) and the Niemann-Pick C1 Like1 protein (NPC1L1) play important roles in cholesterol homeostasis. Both genes encode proteins that are expressed in the intestine and regulate cholesterol absorption [3-5]. However, cholesterol absorption and synthesis are not only determined by genetic factors but also by the metabolic state [6-10]. For example, subjects with high body mass index display high synthesis and low absorption of cholesterol [6-8].
Furthermore, cholesterol synthesis prevails over cholesterol absorption in insulin resistance and type 2 diabetes [711]. In agreement, visceral obesity is associated with a high synthesis phenotype $[12,13]$. Recently, fatty liver, which is thought to be involved in the pathogenesis of the metabolic syndrome [14-17], was also found to be associated with high cholesterol synthesis and low cholesterol absorption [18].

The present work aimed to investigate whether visceral and liver fat contents are correlated with cholesterol homeostasis in healthy humans. Our hypothesis was that even modest differences of liver and visceral fat content would be reflected by differences in cholesterol synthesis and absorption. To answer this question, we performed cross-sectional analyses in 20 healthy individuals who participated in a 
four-week dietary intervention (either very-high-fructose or very-high-glucose diet) study [19]. Another objective of this study was to investigate the impact of very-high-fructose intake, which has been found to alter lipid metabolism [2023], on cholesterol homeostasis.

Visceral and liver fat contents were measured with magnetic resonance (MR) imaging and ${ }^{1} \mathrm{H}-\mathrm{MR}$ spectroscopy, respectively. To estimate cholesterol absorption and synthesis, we measured the serum concentrations of lathosterol, lanosterol, desmosterol (cholesterol precursors, indicate endogenous cholesterol synthesis), campesterol, sitosterol (plant sterols, indicate intestinal cholesterol uptake), and cholestanol (5- $\alpha$ saturated derivative of cholesterol indicates intestinal cholesterol uptake) [24-26].

\section{Methods}

2.1. Study Design and Diet. We report on an exploratory, prospective, randomized, single-blinded, outpatient, intervention study (TUbingen FRuctose Or Glucose study) [19]. Inclusion criteria were age $20-50$ years, body mass index $20-35 \mathrm{~kg} / \mathrm{m}^{2}$, physical health, and not more than onehour sports per week. Exclusion criteria were pregnancy, any relevant illness, fructose intolerance, medication, metal implants, regular alcohol consumption $\geq 10 \mathrm{~g} /$ day, and claustrophobia. The participants received $150 \mathrm{~g}(600 \mathrm{kcal})$ of either fructose or glucose per day for four weeks. They were blinded to the type of intervention. The sugar was provided in identical plastic packs of $50 \mathrm{~g}$ and had to be dissolved in water ( $50 \mathrm{~g}$ sugar in $250 \mathrm{~mL}$ water). The participants were instructed to consume the sugar in addition to a balanced weight-maintaining diet (50\% carbohydrates, 35\% fat, and $15 \%$ protein). Fructose or glucose was ingested three times a day (morning, midday, evening) with the main meals. Dietary counseling was provided by a trained dietitian according to the guidelines of the German Society of Nutrition. We aimed to assess compliance with the dietary prescription by close telephone contact. The participants were instructed to immediately inform the investigators in case of problems with the intake of fructose or glucose. For that, they were provided a calling card. Furthermore, compliance was evaluated by interview at visits 1 and 2 . In addition, the subjects were asked to fill out food intake records on 3 days in each week of the study which were controlled and evaluated by a trained dietician using DGE PC software. Blood sampling, oral glucose tolerance testing, magnetic resonance imaging, and magnetic resonance spectroscopy were performed before and after dietary intervention. The study was approved by the local ethics committee and was conducted in accordance with the "Declaration of Helsinki." Informed written consent was obtained from all participants. Data from the 20 participants who completed the study were included in the present analyses [19].

2.2. Laboratory Analyses. Total, HDL, and LDL cholesterol concentrations were measured with a standard colorimetric method on a Bayer analyzer (Bayer Health Care, Leverkusen, Germany). The serum noncholesterol sterols were measured using gas-liquid chromatography-mass spectrometry-selected ion monitoring (Hewlett Packard 5890) with an automatic injection system (Hewlett Packard Automatic Sampler 7673A) as previously described [27]. Blood glucose was de-termined using a bedside glucose analyzer based on a glucose-oxidase method (Yellow Springs Instruments, Yellow Springs, Colo). Insulin was analyzed by microparticle enzyme immunoassay (Abbott Laboratories, Tokyo, Japan).

2.3. Oral Glucose Tolerance Test. We performed standard $75 \mathrm{~g}$ oral gluose tolerance tests after a 10 -h overnight fast. Venous plasma samples were obtained at $0,30,60,90$, and $120 \mathrm{~min}$ for determination of plasma glucose and insulin. Insulin sensitivity was estimated from the OGTT as proposed by Matsuda and DeFronzo: ISI $_{\text {est }}=10,000 / \sqrt{ }\left(\right.$ Ins $_{\text {mean }} \times$ Gluc $_{\text {mean }} \times$ Ins $_{0} \times$ Gluc $_{0}$ ) [28].

2.4. Quantitative Analysis of Visceral and Liver Fat. Visceral fat mass was measured with an axial T1-weighed fast spin echo technique with a $1.5 \mathrm{~T}$ whole-body imager (Magnetom Sonata; Siemens Medical Solutions) in the complete abdominal region, ranging from head of femur to head of humerus [29]. Liver fat was determined by localized proton magnetic resonance spectroscopy applying a single-voxel STEAM technique with short echo time (TE) as previously described $[30,31]$.

2.5. Statistical Analysis. The clinical and biochemical characteristics are presented as numbers and percentages and means \pm standard errors of the means for categorical and continuous data, respectively. Ratios of the noncholesterol sterols to cholesterol (measured with gas-liquid chromatography) were calculated (see Table 2). The univariate relationships of the noncholesterol sterols with cholesterol, the relationships among the noncholesterol sterol to cholesterol ratios, and the relationships of the cholesterol subfractions and the noncholesterol sterol ratios with fat depots and insulin sensitivity were analyzed with linear regression models. The results are shown as Pearson correlation coefficients. Furthermore, we performed multivariate analysis for the associations of the cholesterol subfractions and the noncholesterol sterol to cholesterol ratios with fat depots and insulin sensitivity using Analysis of Covariance (ANCOVA). Alterations in the noncholesterol sterol to cholesterol ratios in response to fructose and glucose intervention were studied with the paired samples $t$-test (two-sided tests). ANCOVA was used to compare the changes in the noncholesterol sterol to cholesterol ratios (e.g., change in lathosterol to cholesterol ratio between baseline and followup examination) between the fructose and glucose intervention groups, with study group as the main factor and the metabolic parameter of interest at baseline (e.g., lathosterol to cholesterol ratio at baseline examination) as covariate (two-sided tests). To estimate the treatment effect, differences in least-square means and the corresponding 95\% confidence intervals were calculated based on the ANCOVA models [32]. Data that were not normally distributed (Shapiro-Wilk $W$ test) were 
TABLE 1: Baseline characteristics of the study participants.

\begin{tabular}{lc}
\hline & Baseline examination \\
\hline Males/females, $n$ & $12 / 8$ \\
Age, years & $30.5 \pm 2.0$ \\
Body mass index, $\mathrm{kg} / \mathrm{m}^{2}$ & $25.9 \pm 0.5$ \\
Waist, cm & $85 \pm 2$ \\
Visceral fat, kg & $2.2 \pm 0.2$ \\
Liver fat, \% signal & $1.5 \pm 0.2$ \\
Systolic blood pressure, mmHg & $117 \pm 3$ \\
Diastolic blood pressure, mmHg & $77 \pm 2$ \\
Total cholesterol, mg/dL & $175 \pm 5$ \\
LDL cholesterol, mg/dL & $106 \pm 5$ \\
HDL cholesterol, mg/dL & $54 \pm 2$ \\
VLDL cholesterol, mg/dL & $15 \pm 2$ \\
Non-HDL cholesterol, mg/dL & $122 \pm 5$ \\
Fasting glucose, mmol/L & $4.86 \pm 0.06$ \\
Fasting insulin, pmol/L & $48 \pm 7$ \\
Insulin sensitivity Matsuda, & $17.6 \pm 2.1$ \\
arbitrary units &
\end{tabular}

Values are numbers and percentages and means with standard errors of the means for categorical and continuous data, respectively.

TABLE 2: Serum levels of the noncholesterol sterol to cholesterol ratios at baseline.

\begin{tabular}{ll}
\hline Lathosterol/cholesterol, $\mu \mathrm{g} / \mathrm{mg}$ & $1.28 \pm 0.11$ \\
Desmosterol/cholesterol, $\mu \mathrm{g} / \mathrm{mg}$ & $0.49 \pm 0.02$ \\
Lanosterol/cholesterol, $\mu \mathrm{g} / \mathrm{mg}$ & $0.31 \pm 0.02$ \\
Campesterol/cholesterol, $\mu \mathrm{g} / \mathrm{mg}$ & $1.58 \pm 0.11$ \\
Sitosterol/cholesterol, $\mu \mathrm{g} / \mathrm{mg}$ & $1.22 \pm 0.09$ \\
Cholestanol/cholesterol, $\mu \mathrm{g} / \mathrm{mg}$ & $1.72 \pm 0.06$ \\
\hline
\end{tabular}

Values are means with standard errors of the means.

transformed logarithmically (base-10). $P$ values $<0.05$ were considered significant. The JMP statistical software package 7.0 (SAS Institute, Cary, NC, USA) was used.

\section{Results}

The baseline characteristics of the study participants are shown in Table 1. The mean \pm standard error of the mean serum concentrations were $186 \pm 5 \mathrm{mg} / \mathrm{dL}$ for cholesterol (GCMS), $0.241 \pm 0.024 \mathrm{mg} / \mathrm{dL}$ for lathosterol, $0.091 \pm$ $0.006 \mathrm{mg} / \mathrm{dL}$ for desmosterol, $0.058 \pm 0.004 \mathrm{mg} / \mathrm{dL}$ for lanosterol, $0.295 \pm 0.023 \mathrm{mg} / \mathrm{dL}$ for campesterol, $0.229 \pm$ $0.018 \mathrm{mg} / \mathrm{dL}$ for sitosterol, and $0.320 \pm 0.014 \mathrm{mg} / \mathrm{dL}$ for cholestanol.

The serum desmosterol and cholestanol levels were significantly related to cholesterol $(r=0.631, P=.002$ and $r=0.615, P=.004$, resp.). The lathosterol, desmosterol, and lanosterol to cholesterol ratios were also positively correlated (Table 3). In agreement, the ratios of campesterol and sitosterol to cholesterol showed a significant positive association (Table 3). Furthermore, the ratio of campesterol

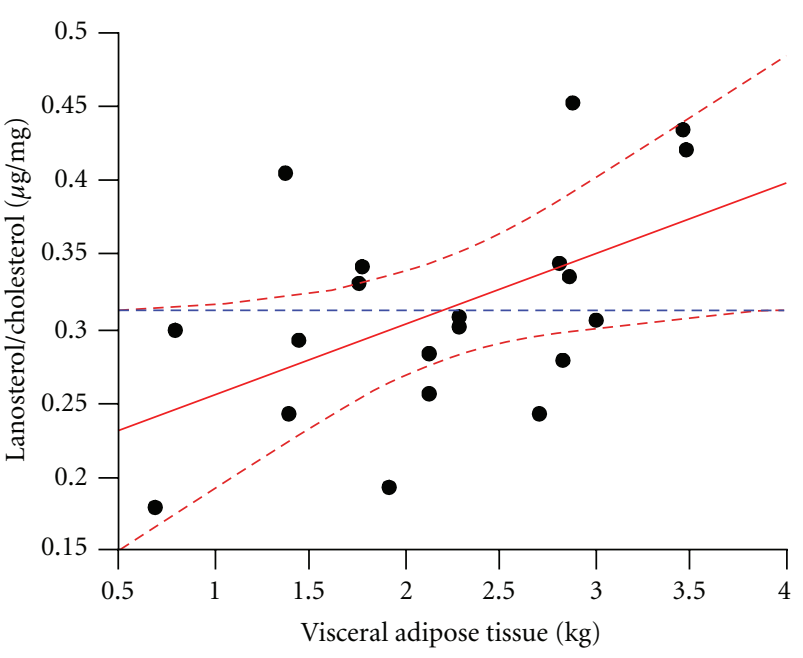

(a)

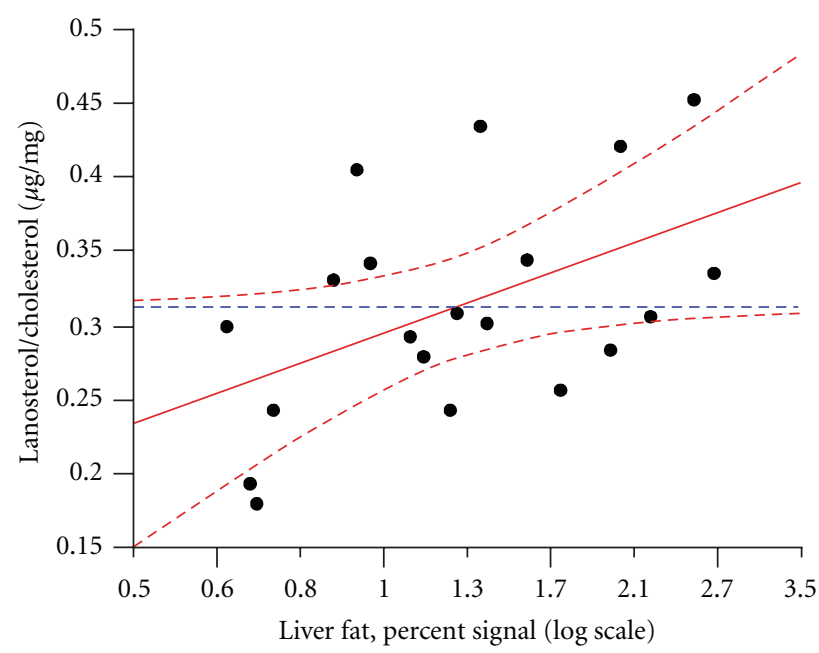

(b)

Figure 1: The associations of (a) visceral and (b) liver fat with the lanosterol to cholesterol ratio adjusted for sex, age, and body mass index.

to cholesterol was significantly related to the ratio of cholestanol to cholesterol (Table 3). The ratio of lathosterol to cholesterol was inversely related to the ratio of campesterol to cholesterol (Table 3).

High lanosterol and desmosterol to cholesterol ratios were significantly associated with increased visceral and liver fat content (Table 4). The association of the lanosterol to cholesterol ratio with visceral $(P=.033)$ and liver fat $(P=.044)$ was independent of sex, age, and body mass index (Figure 1). The cholesterol absorption markers were not significantly related to visceral and liver fat (Table 4). HDL cholesterol was inversely related to visceral fat and liver fat content whereas non-HDL cholesterol was positively correlated with visceral fat (Table 4). LDL cholesterol was not associated with fat depots (Table 4). The lathosterol to cholesterol ratio was inversely related to insulin sensitivity (Table 4).

The lathosterol to cholesterol ratio significantly decreased in response to very-high-fructose diet but not in 
TABLE 3: Univariate correlations among the noncholesterol sterol to cholesterol ratios.

\begin{tabular}{lcccccc}
\hline & $\begin{array}{c}\text { Lathosterol/ } \\
\text { cholesterol }\end{array}$ & $\begin{array}{c}\text { Desmosterol/ } \\
\text { cholesterol }\end{array}$ & $\begin{array}{c}\text { Lanosterol/ } \\
\text { cholesterol }\end{array}$ & $\begin{array}{c}\text { Campesterol/ } \\
\text { cholesterol }\end{array}$ & $\begin{array}{c}\text { Sitosterol/ } \\
\text { cholesterol }\end{array}$ & $\begin{array}{c}\text { Cholestanol/ } \\
\text { cholesterol }\end{array}$ \\
\hline Lathosterol/cholesterol & - & $0.572^{\dagger}$ & $0.489^{\ddagger}$ & $-0.493^{\ddagger}$ & -0.378 & -0.378 \\
Desmosterol/cholesterol & $0.572^{\dagger}$ & - & $0.607^{\dagger}$ & -0.280 & -0.173 & -0.357 \\
Lanosterol/cholesterol & $0.489^{\ddagger}$ & $0.607^{\dagger}$ & - & -0.059 & 0.000 & -0.036 \\
Campesterol/cholesterol & $-0.493^{\ddagger}$ & -0.280 & -0.059 & - & $0.880^{*}$ & $0.486^{\ddagger}$ \\
Sitosterol/cholesterol & -0.378 & -0.173 & 0.000 & - & 0.375 \\
Cholestanol/cholesterol & -0.378 & -0.357 & -0.036 & $0.486^{\ddagger}$ & - & - \\
\hline
\end{tabular}

Values are Pearson correlation coefficients calculated with linear regression; ${ }^{*} P<0.001,{ }^{\dagger} P<0.01,{ }^{\ddagger} P<0.05$.

TABLE 4: Univariate correlations of total cholesterol, cholesterol subfractions, and the noncholesterol sterol to cholesterol ratios with liver fat, visceral fat, and insulin sensitivity.

\begin{tabular}{|c|c|c|c|c|c|c|}
\hline & \multicolumn{2}{|c|}{ Liver fat } & \multicolumn{2}{|c|}{ Visceral fat } & \multicolumn{2}{|c|}{ Insulin sensitivity } \\
\hline & $r$ & $P$ & $r$ & $P$ & $r$ & $P$ \\
\hline Total cholesterol & 0.194 & 0.384 & 0.243 & 0.303 & 0.082 & 0.730 \\
\hline LDL cholesterol & 0.386 & 0.145 & 0.363 & 0.116 & -0.049 & 0.839 \\
\hline HDL cholesterol & -0.545 & 0.011 & -0.593 & 0.006 & 0.045 & 0.852 \\
\hline VLDL cholesterol & 0.035 & 0.884 & 0.254 & 0.279 & 0.280 & 0.231 \\
\hline Non-HDL cholesterol & 0.386 & 0.093 & 0.452 & 0.046 & 0.066 & 0.783 \\
\hline Lathosterol/cholesterol & 0.107 & 0.636 & 0.404 & 0.078 & -0.500 & 0.025 \\
\hline Desmosterol/cholesterol & 0.548 & 0.027 & 0.541 & 0.014 & -0.259 & 0.271 \\
\hline Lanosterol/cholesterol & 0.642 & 0.004 & 0.629 & 0.003 & -0.364 & 0.114 \\
\hline Campesterol/cholesterol & -0.005 & 0.710 & -0.253 & 0.282 & 0.335 & 0.149 \\
\hline Sitosterol/cholesterol & 0.040 & 0.803 & -0.279 & 0.234 & 0.200 & 0.397 \\
\hline Cholestanol/cholesterol & 0.008 & 0.974 & 0.060 & 0.800 & 0.325 & 0.163 \\
\hline
\end{tabular}

$r$ Pearson correlation coefficients and $P$ values calculated with linear regression (liver fat was transformed logarithmically for calculation of the $P$ values).

response to very-high-glucose diet with the difference between interventions reaching statistical significance (Table 5). In agreement, there was a significant treatment effect for the alterations of the lanosterol to cholesterol ratio (Table 5). No changes or treatment effects were found for the desmosterol to cholesterol ratio and the absorption marker to cholesterol ratios (Table 5).

\section{Discussion}

We found that the ratios of desmosterol and lanosterol to cholesterol, which indicate endogenous cholesterol synthesis, were positively associated with visceral and liver fat content in persons at relatively low metabolic risk. The relationships of the lanosterol to cholesterol ratio with visceral and liver fat were independent of obesity. Furthermore, high ratio of lathosterol to cholesterol, also an indicator of cholesterol synthesis, was related to lower insulin sensitivity.

Our observations fit well in the context of the previously published studies in the field. High body mass index, insulin resistance, and type 2 diabetes have been independently associated with high cholesterol synthesis [6-11]. Visceral obesity and just recently fatty liver were also related to increased synthesis of cholesterol [12, 13, 18]. Our study extends these findings in the sense that we report on a cohort of healthy people. Furthermore, we have also measured the cholesterol synthesis marker lanosterol which was obviously the noncholesterol sterol most strongly related to visceral and liver fat content. We did not find significant associations of cholesterol absorption with visceral or liver fat. This may suggest that fat distribution and ectopic fat deposition in the liver primarily affect cholesterol synthesis. In this respect, it seems noteworthy that visceral fat was not significantly related to the sitosterol to cholesterol ratio in a recent study either [13].

Why is liver fat content positively correlated with cholesterol synthesis? The most important regulator of cellular cholesterol synthesis is the sterol regulatory element-binding protein 2 (SREBP2), a membrane-bound transcription factor $[33,34]$. This transcription factor is highly expressed in the liver and interestingly, its activity is increased in subjects with high liver fat [35]. However, the exact mechanisms accounting for the activation of SREBP2 in subjects with high liver fat seem poorly understood.

Since cholesterol homeostasis is obviously associated with liver fat content, the following question arises: might pharmacological interventions targeting cholesterol homeostasis have an impact on hepatic lipid content? A wellperformed study by Szendroedi et al. found that even highdose simvastatin treatment has no direct effects on liver fat 
TABLE 5: Changes in the noncholesterol sterol to cholesterol ratios in response to high-fructose or high-glucose diet.

\begin{tabular}{lcccccccccc}
\hline & \multicolumn{3}{c}{ Fructose intervention group } & \multicolumn{3}{c}{ Glucose intervention group } & \multicolumn{3}{c}{ Fructose versus glucose } \\
& Baseline & Change & $P^{*}$ & Baseline & Change & $P^{*}$ & $\Delta$ LSM & $95 \%$ CI & $P^{\dagger}$ \\
\hline Lathosterol/cholesterol, $\mu \mathrm{g} / \mathrm{mg}$ & $1.16 \pm 0.11$ & $-0.20 \pm 0.06$ & 0.006 & $1.40 \pm 0.20$ & $-0.08 \pm 0.18$ & 0.659 & -0.28 & -0.53 to -0.04 & 0.027 \\
Desmosterol/cholesterol, $\mu \mathrm{g} / \mathrm{mg}$ & $0.45 \pm 0.03$ & $0.01 \pm 0.02$ & 0.675 & $0.52 \pm 0.04$ & $-0.02 \pm 0.04$ & 0.638 & -0.01 & -0.08 to 0.07 & 0.809 \\
Lanosterol/cholesterol, $\mu \mathrm{g} / \mathrm{mg}$ & $0.30 \pm 0.03$ & $-0.03 \pm 0.03$ & 0.332 & $0.32 \pm 0.02$ & $0.03 \pm 0.04$ & 0.555 & -0.08 & -0.16 to 0.00 & 0.040 \\
Campesterol/cholesterol, $\mu \mathrm{g} / \mathrm{mg}$ & $1.74 \pm 0.13$ & $-0.07 \pm 0.07$ & 0.337 & $1.42 \pm 0.17$ & $0.05 \pm 0.15$ & 0.746 & -0.10 & -0.47 to 0.27 & 0.569 \\
Sitosterol/cholesterol, $\mu \mathrm{g} / \mathrm{mg}$ & $1.28 \pm 0.09$ & $-0.07 \pm 0.05$ & 0.165 & $1.16 \pm 0.15$ & $0.01 \pm 0.09$ & 0.889 & -0.06 & -0.26 to 0.14 & 0.562 \\
Cholestanol/cholesterol, $\mu \mathrm{g} / \mathrm{mg}$ & $1.82 \pm 0.06$ & $-0.10 \pm 0.10$ & 0.348 & $1.61 \pm 0.09$ & $-0.07 \pm 0.06$ & 0.307 & -0.04 & -0.32 to 0.25 & 0.796 \\
\hline
\end{tabular}

Values are means \pm standard errors of the means; change: absolute difference between visits 1 and 2; fructose versus glucose: treatment effect of fructose intervention compared to glucose intervention; $\triangle \mathrm{LSM}$ : difference in least squares means between fructose and glucose intervention (calculated with Analysis of Covariance with correction for baseline values); CI: confidence interval; ${ }^{*} P$ value for change between visits 1 and 2 calculated with paired samples $t$-test (two-sided); ${ }^{\dagger} P$ value for difference in change between fructose and glucose intervention (calculated with Analysis of Covariance with correction for baseline values, two-sided).

content in people with type 2 diabetes [36]. In contrast, the cholesterol absorption inhibitor ezetimibe was found to increase the reduction of liver fat in obese subjects on a weight-loss diet [37]. Whether the use of plant sterols and stanols, which similarly act as inhibitors of cholesterol absorption [38], will help to reduce hepatic steatosis remains to be investigated.

We also studied the effects of very high-fructose and veryhigh-glucose diets on cholesterol absorption and synthesis. High-fructose diet has been implicated in the pathogenesis of the metabolic syndrome, fatty liver, and type 2 diabetes [ $20-$ 23]. Moreover, a recent study suggested that high-fructose diet was associated with increased plasma concentrations of LDL cholesterol, small dense LDL, and oxidized LDL [23]. The effect of high-fructose diet on cholesterol homostasis has not been investigated so far. According to the present findings, fructose compared with glucose appears to less strongly stimulate cholesterol synthesis. This novel observation may be explained by the fact that fructose does not provoke endogenous secretion of insulin [20], which is considered to be an important regulator of cholesterol synthesis [39]. Alternatively, the treatment effect for cholesterol synthesis may result from the significant weight gain in the glucose intervention group $(+1.7 \mathrm{~kg})$ which was not observed in the fructose intervention group $(+0.2 \mathrm{~kg})$ [19].

Consistent with earlier work [12, 13, 40,41], the serum HDL cholesterol concentration was significantly decreased in subjects with high visceral and liver fat content in the present cohort. Hence, increased visceral and liver fat content may indicate early disturbance of lipid metabolism in healthy people. It is also in agreement with a recent trial that the serum total and LDL cholesterol concentrations were not significantly related to visceral and liver fat content in our cohort of healthy individuals [12]. Hoenig et al. even showed that low-density lipoprotein cholesterol was inversely correlated with the abdominal visceral fat area in subjects with established vascular disease [13]. The authors discussed that their finding could explain the loss of the relationship between LDL cholesterol and cardiovascular events in the obese and support the use of non-HDL cholesterol instead of LDL cholesterol as the primary therapeutic target for lipid lowering therapy $[13,42]$. Our data may support this view considering that we observed a positive correlation of nonHDL cholesterol with visceral fat.

Finally, our data confirm that intestinal cholesterol absorption and endogenous cholesterol synthesis are interrelated considering the significant inverse association between the ratios of lathosterol and campesterol to cholesterol [7, 24].

The sample size of our study is relatively low. We cannot, therefore, rule out that a significant association of visceral and liver fat content with the cholesterol absorption markers could be observed in a larger cohort of healthy individuals. To compensate for this drawback, we used very precise and stringently validated analytical procedures for the quantification of the noncholesterol sterols and the fat depots. The serum concentrations of the noncholesterol sterols were measured using a highly sensitive and specific gas-liquid chromatography method. Visceral and liver fat content were quantified using magnetic resonance imaging and magnetic resonance spectroscopy, respectively. We also want to highlight that the sample size of our cohort was similar or even larger compared with previous highly recognized studies fructose intervention studies [21].

In conclusion, we found an independent association of visceral and liver fat content with cholesterol synthesis in healthy humans. Moreover, we were able to show for the first time that cholesterol synthesis is dependent on fructose/glucose intake. Studies investigating whether marked alterations of liver fat content will have an impact on cholesterol homeostasis are encouraged.

\section{Author Contributions}

A. Fritsche, G. Silbernagel, and N. Stefan designed the study. D. Lütjohann and S. Meichsner measured the noncholesterol sterols. J. Machann and F. Schick quantified the fat depots. G. Silbernagel performed the statistical analysis. A. Fritsche, D. Lütjohann, G. Silbernagel, and N. Stefan wrote the manuscript. K. Kantartzis contributed to the discussion and reviewed and edited the manuscript. All authors have read the final version and agreed to the manuscript as submitted. 


\section{Conflict of Interest}

The authors have no conflict of interest to declare.

\section{Acknowledgments}

The authors thank all study participants for their cooperation. Furthermore, they gratefully acknowledge the help and excellent technical assistance of A. Bury, M. Graf, B. Horrer, A. Kerksiek, E. Kollmar, S. Kümmerle, V. Schumacher, S. Unmuth, and A. Vosseler. The study was supported by a grant from the German Research Foundation (KFO 114/2) and a grant (Grant no. 4 AI) from the Zentrum Ernährungsmedizin Tübingen-Hohenheim. Funding did not include industrial sponsorship.

\section{References}

[1] K. von Bergmann, T. Sudhop, and D. Lütjohann, "Cholesterol and plant sterol absorption: recent insights," American Journal of Cardiology, vol. 96, no. 1, pp. 10D-14D, 2005.

[2] O. Weingärtner, D. Lütjohann, M. Böhm, and U. Laufs, "Relationship between cholesterol synthesis and intestinal absorption is associated with cardiovascular risk," Atherosclerosis, vol. 210, no. 2, pp. 362-365, 2010.

[3] K. E. Berge, H. Tian, G. A. Graf et al., "Accumulation of dietary cholesterol in sitosterolemia caused by mutations in adjacent ABC transporters," Science, vol. 290, no. 5497, pp. 1771-1775, 2000.

[4] M. H. Lee, K. Lu, S. Hazard et al., "Identification of a gene, ABCG5, important in the regulation of dietary cholesterol absorption," Nature Genetics, vol. 27, no. 1, pp. 79-83, 2001.

[5] S. W. Altmann, H. R. Davis Jr., L. J. Zhu et al., "Niemannpick $\mathrm{C} 1$ like 1 protein is critical for intestinal cholesterol absorption," Science, vol. 303, no. 5661, pp. 1201-1204, 2004.

[6] P. Simonen, H. Gylling, A. N. Howard, and T. A. Miettinen, "Introducing a new component of the metabolic syndrome: low cholesterol absorption," American Journal of Clinical Nutrition, vol. 72, no. 1, pp. 82-88, 2000.

[7] G. Silbernagel, G. Fauler, M. M. Hoffmann et al., "The associations of cholesterol metabolism and plasma plant sterols with all-cause and cardiovascular mortality," Journal of Lipid Research, vol. 51, no. 8, pp. 2384-2393, 2010.

[8] G. Silbernagel, G. Fauler, W. Renner et al., "The relationships of cholesterol metabolism and plasma plant sterols with the severity of coronary artery disease," Journal of Lipid Research, vol. 50, no. 2, pp. 334-341, 2009.

[9] J. Pihlajamäki, H. Gylling, T. A. Miettinen, and M. Laakso, "Insulin resistance is associated with increased cholesterol synthesis and decreased cholesterol absorption in normoglycemic men," Journal of Lipid Research, vol. 45, no. 3, pp. 507-512, 2004.

[10] P. P. Simonen, H. K. Gylling, and T. A. Miettinen, "Diabetes contributes to cholesterol metabolism regardless of obesity," Diabetes Care, vol. 25, no. 9, pp. 1511-1515, 2002.

[11] M. R. Hoenig and F. W. Sellke, "Insulin resistance is associated with increased cholesterol synthesis, decreased cholesterol absorption and enhanced lipid response to statin therapy," Atherosclerosis, vol. 211, no. 1, pp. 260-265, 2010.

[12] P. Peltola, J. Pihlajamäki, H. Koutnikova et al., "Visceral obesity is associated with high levels of serum squalene," Obesity, vol. 14, no. 7, pp. 1155-1163, 2006.
[13] M. R. Hoenig, G. Cowin, R. Buckley, C. McHenery, and A. Coulthard, "Low density lipoprotein cholesterol is inversely correlated with abdominal visceral fat area: a magnetic resonance imaging study," Lipids in Health and Disease, vol. 10, article 12, 2011.

[14] N. Stefan, K. Kantartzis, and H. U. Häring, "Causes and metabolic consequences of fatty liver," Endocrine Reviews, vol. 29, no. 7, pp. 939-960, 2008.

[15] E. Fabbrini, F. Magkos, B. S. Mohammed et al., "Intrahepatic fat, not visceral fat, is linked with metabolic complications of obesity," Proceedings of the National Academy of Sciences of the United States of America, vol. 106, no. 36, pp. 15430-15435, 2009.

[16] J. C. Cohen, J. D. Horton, and H. H. Hobbs, "Human fatty liver disease: old questions and new insights," Science, vol. 332, no. 6037, pp. 1519-1523, 2011.

[17] N. Stefan and H. U. Haring, "The metabolically benign and malignant fatty liver," Diabetes, vol. 60, no. 8, pp. 2011-2017, 2011.

[18] P. Simonen, A. Kotronen, M. Hallikainen et al., "Cholesterol synthesis is increased and absorption decreased in nonalcoholic fatty liver disease independent of obesity," Journal of Hepatology, vol. 54, no. 1, pp. 153-159, 2011.

[19] G. Silbernagel, J. Machann, S. Unmuth et al., "Effects of 4week very-high-fructose/glucose diets on insulin sensitivity, visceral fat and intrahepatic lipids: an exploratory trial," British Journal of Nutrition, vol. 106, no. 1, pp. 79-86, 2011.

[20] L. Tappy and K. A. Lê, "Metabolic effects of fructose and the worldwide increase in obesity," Physiological Reviews, vol. 90, no. 1, pp. 23-46, 2010.

[21] K. A. Lê, D. Faeh, R. Stettler et al., "A 4-wk high-fructose diet alters lipid metabolism without affecting insulin sensitivity or ectopic lipids in healthy humans," American Journal of Clinical Nutrition, vol. 84, no. 6, pp. 1374-1379, 2006.

[22] K. A. Lê, M. Ith, R. Kreis et al., "Fructose overconsumption causes dyslipidemia and ectopic lipid deposition in healthy subjects with and without a family history of type 2 diabetes," American Journal of Clinical Nutrition, vol. 89, no. 6, pp. 17601765, 2009.

[23] K. L. Stanhope, J. M. Schwarz, N. L. Keim et al., "Consuming fructose-sweetened, not glucose-sweetened, beverages increases visceral adiposity and lipids and decreases insulin sensitivity in overweight/obese humans," Journal of Clinical Investigation, vol. 119, no. 5, pp. 1322-1334, 2009.

[24] T. A. Miettinen, R. S. Tilvis, and Y. A. Kesaniemi, "Serum plant sterols and cholesterol precursors reflect cholesterol absorption and synthesis in volunteers of a randomly selected male population," American Journal of Epidemiology, vol. 131, no. 1, pp. 20-31, 1990.

[25] N. R. Matthan and A. H. Lichtenstein, "Approaches to measuring cholesterol absorption in humans," Atherosclerosis, vol. 174, no. 2, pp. 197-205, 2004.

[26] G. Silbernagel and W. März, "Plant sterols: cardiovascular risk factors?" Journal of Laboratory and Clinical Medicine, vol. 32, no. 4, pp. 209-218, 2008.

[27] O. Weingärtner, T. Pinsdorf, K. S. Rogacev et al., "The relationships of markers of cholesterol homeostasis with carotid intima-media thickness," PLoS One, vol. 5, no. 10, Article ID e13467, 2010.

[28] M. Matsuda and R. A. DeFronzo, "Insulin sensitivity indices obtained from oral glucose tolerance testing: comparison with the euglycemic insulin clamp," Diabetes Care, vol. 22, no. 9, pp. 1462-1470, 1999. 
[29] J. Machann, C. Thamer, B. Schnoedt et al., "Standardized assessment of whole body adipose tissue topography by MRI," Journal of Magnetic Resonance Imaging, vol. 21, no. 4, pp. 455462, 2005.

[30] E. Fabbrini, C. Conte, and F. Magkos, "Methods for assessing intrahepatic fat content and steatosis," Current Opinion in Clinical Nutrition and Metabolic Care, vol. 12, no. 5, pp. 474481, 2009.

[31] N. F. Schwenzer, F. Springer, C. Schraml, N. Stefan, J. Machann, and F. Schick, "Non-invasive assessment and quantification of liver steatosis by ultrasound, computed tomography and magnetic resonance," Journal of Hepatology, vol. 51, no. 3, pp. 433-445, 2009.

[32] K. F. Schulz, D. G. Altman, and D. Moher, "CONSORT 2010 statement: updated guidelines for reporting parallel group randomised trials," British Medical Journal, vol. 340, article 32, p. c332, 2010.

[33] M. S. Brown and J. L. Goldstein, "The SREBP pathway: regulation of cholesterol metabolism by proteolysis of a membranebound transcription factor," Cell, vol. 89, no. 3, pp. 331-340, 1997.

[34] J. D. Horton, J. L. Goldstein, and M. S. Brown, "SREBPs: activators of the complete program of cholesterol and fatty acid synthesis in the liver," Journal of Clinical Investigation, vol. 109, no. 9, pp. 1125-1131, 2002.

[35] F. Caballero, A. Fernández, A. M. De Lacy, J. C. FernándezCheca, J. Caballería, and C. García-Ruiz, "Enhanced free cholesterol, SREBP-2 and StAR expression in human NASH," Journal of Hepatology, vol. 50, no. 4, pp. 789-796, 2009.

[36] J. Szendroedi, C. Anderwald, M. Krssak et al., "Effects of highdose simvastatin therapy on glucose metabolism and ectopic lipid deposition in nonobese type 2 diabetic patients," Diabetes Care, vol. 32, no. 2, pp. 209-214, 2009.

[37] D. C. Chan, G. F. Watts, S. K. Gan, E. M. Ooi, and P. H. Barrett, "Effect of ezetimibe on hepatic fat, inflammatory markers, and apolipoprotein B-100 kinetics in insulin-resistant obese subjects on a weight loss diet," Diabetes Care, vol. 33, no. 5, pp. 1134-1139, 2010.

[38] M. B. Katan, S. M. Grundy, P. Jones, M. Law, T. Miettinen, and R. Paoletti, "Efficacy and safety of plant stanols and sterols in the management of blood cholesterol levels," Mayo Clinic Proceedings, vol. 78, no. 8, pp. 965-978, 2003.

[39] K. A. Tobin, S. M. Ulven, G. U. Schuster et al., "Liver $\mathrm{X}$ receptors as insulin-mediating factors in fatty acid and cholesterol biosynthesis," Journal of Biological Chemistry, vol. 277, no. 12, pp. 10691-10697, 2002.

[40] F. Magkos, B. S. Mohammed, and B. Mittendorfer, "Effect of obesity on the plasma lipoprotein subclass profile in normoglycemic and normolipidemic men and women," International Journal of Obesity, vol. 32, no. 11, pp. 1655-1664, 2008.

[41] K. Kantartzis, K. Rittig, A. Cegan et al., "Fatty liver is independently associated with alterations in circulating HDL2 and HDL3 subfractions," Diabetes Care, vol. 31, no. 2, pp. 366-368, 2008.

[42] M. R. Hoenig, "Implications of the obesity epidemic for lipidlowering therapy: non-HDL cholesterol should replace LDL cholesterol as the primary therapeutic target," Vascular Health and Risk Management, vol. 4, no. 1, pp. 143-156, 2008. 


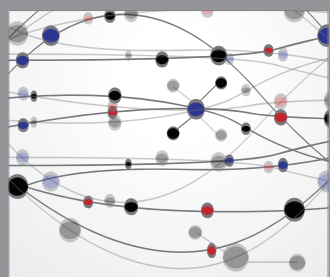

The Scientific World Journal
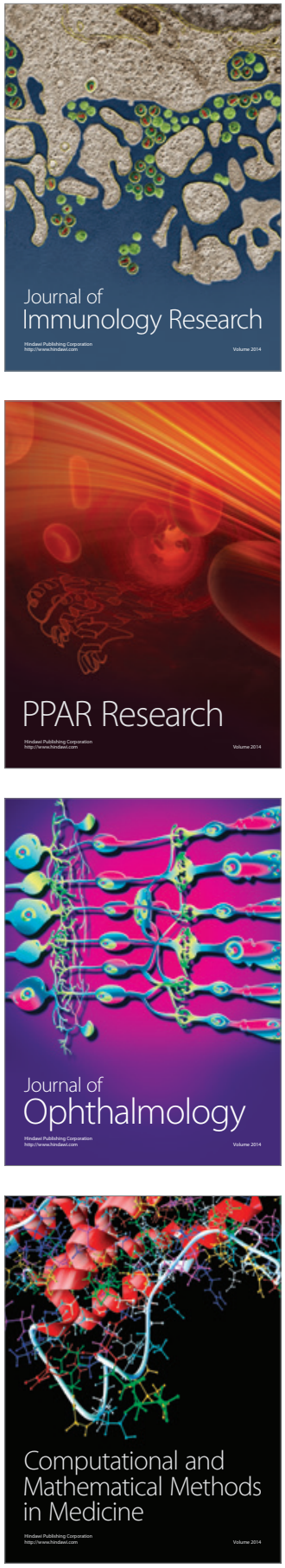

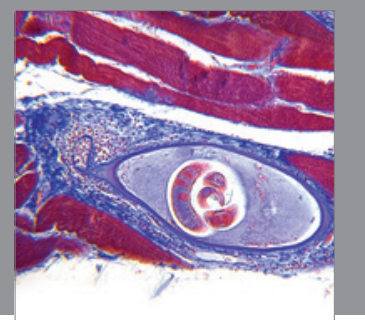

Gastroenterology

Research and Practice
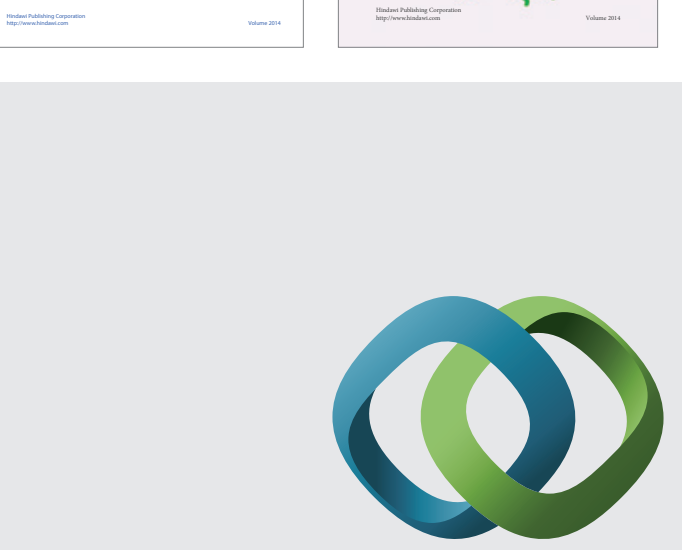

\section{Hindawi}

Submit your manuscripts at

http://www.hindawi.com
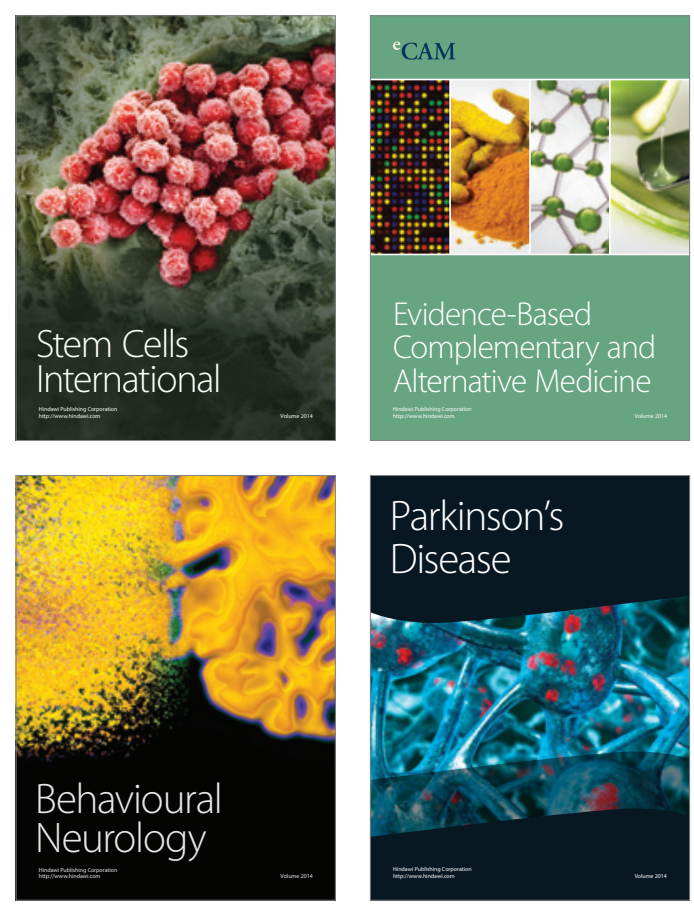

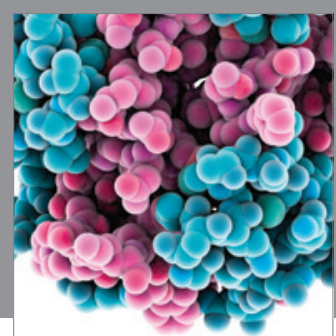

Journal of
Diabetes Research

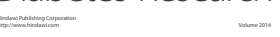

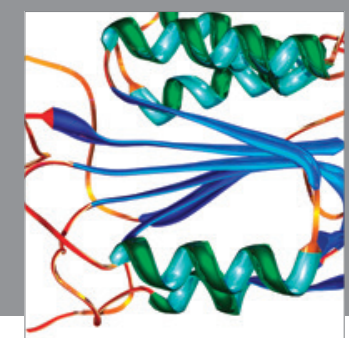

Disease Markers
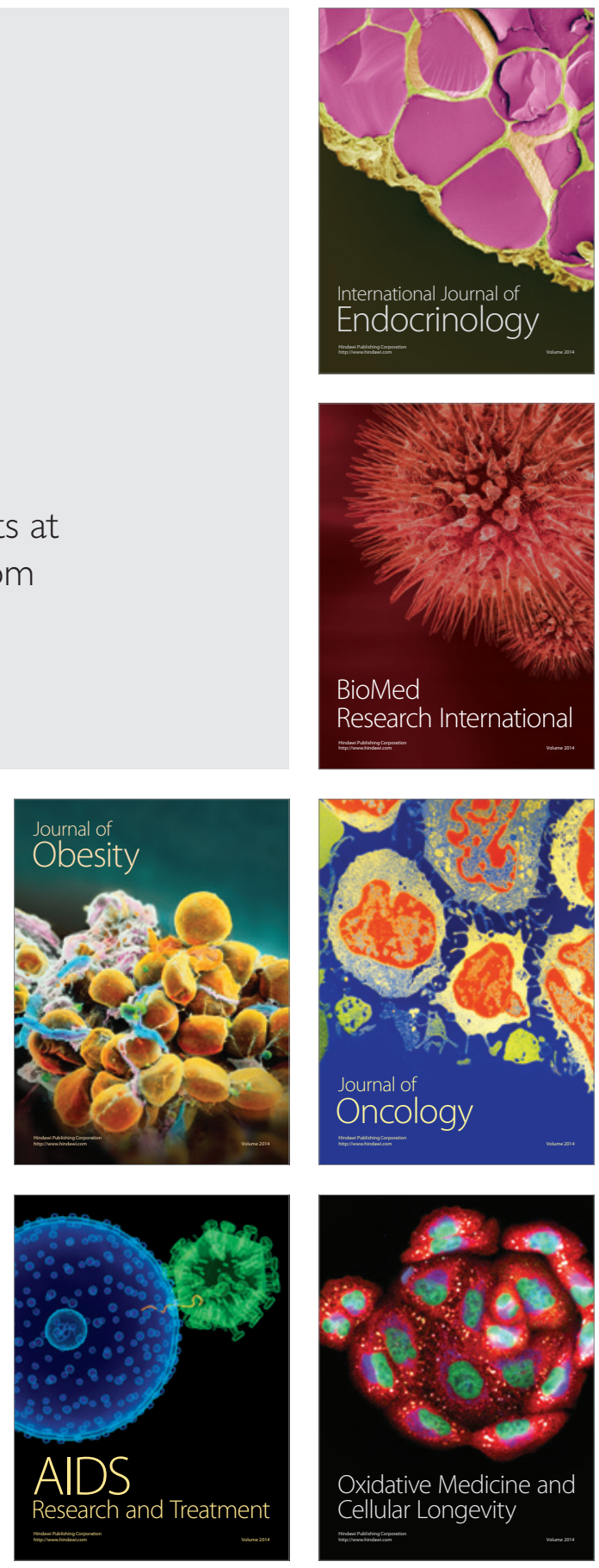